\title{
Toward an Entirely Endovascular World for Thoracoabdominal Aortic Disease: Myth or Reality?
}

\author{
Piergiorgio Cao and Ciro Ferrer
}

\subsection{Introduction}

Endovascular repair (ER) is currently the firstline treatment for the overwhelming majority of aortic disease, and its applicability is expanding to the whole aorta in light of evolving technologies. Whereas with the introduction of new devices developed to overcome common anatomical limitations, techniques of infrarenal (EVAR) and descending thoracic endovascular aneurysm repair (TEVAR) have reached a plateau; the debate is still open on thoracoabdominal aortic aneurysm (TAAA).

Despite continuous intra- and postoperative improvements, open repair (OR) of TAAA is still associated with significant mortality and morbidity, mainly due to the invasiveness of the surgical access and the hemodynamic instability, following proximal aortic clamping and massive blood loss. As the population ages and diagnostic capabilities improve, elderly patients with multiple comorbidities and limited physiological reserve are increasingly referred for treatment of TAAAs. The need to further reduce morbidity and mortality associated with open surgery has made the endovascular approach particularly attractive.

P. Cao $(\bowtie)$

Mater Dei Hospital, Rome, Italy

C. Ferrer

Department of Surgery "Pietro Valdoni”, Sapienza

University of Rome, Rome, Italy
Fenestrated stent grafts were originally developed to extend the area suitable for proximal sealing in short-neck abdominal aortic aneurysms (AAAs). Once it was established that the presence of fenestrations did not jeopardize the integrity of the proximal sealing zone or incorporated arteries, clinicians began to extend repairs to more complex aneurysms using covered stents to bridge fenestrations to target vessels.

However, it soon became evident that a fenestrated solution was not adequate to cope with extensive TAAA in which the luminal diameter within the visceral segment of the aorta was much larger than the aortic stent graft. Thus, the concept of a side-arm branched graft, in which a fabric cuff is attached to the main body, was developed, leaving the fenestrated solutions to cases where the aortic internal lumen does not leave enough room for branched stent graft. Considerable progress has been made over the last few years to refine this technique. The introduction of off-the-shelf devices, which sufficiently met anatomical variability, has partially solved the problem of long manufacturing time of custom-made stent grafts. Parallel grafts, as well, play a complementary role in emergency situations where custom-made devices are not available. Staging of extensive repairs is widely adopted to prevent symptoms of spinal cord ischemia (SCI), as well as the full relining of bridged target vessels with bare-metal stents, in order to increase branch stability. Finally, preloaded systems allow for wider anatomic eligibility for complex aortic repair. 
In this scenario, endovascular repair for thoracoabdominal aortic disease with fenestrated (FEVAR) and branched (BEVAR) stent grafts plays a leading role with excellent short-term results and promising long-term durability.

This chapter focuses on clinical and technical aspects involved in the decision-making for the management of TAAA, endovascular anatomical feasibility, and outcome evaluation and tries to determine whether the concept of an entirely endovascular world for thoracoabdominal aortic disease is conceivable.

\subsection{Endovascular Feasibility}

Total endovascular repair of thoracoabdominal aortic disease depends on the assessment of specific morphological criteria, including landing zones, aortic internal lumen diameter, aortic tortuosity, visceral vessel orientation, and quality of access vessels. Computed tomographic angiography (CTA) of the aorta and branch vessels and three-dimensional reconstruction and postprocessing image manipulation software are essential tools when advanced endovascular techniques are being utilized [1]. Multiplanar reconstructions according to the center lumen line are essential for accurate evaluation and planning, including the assessment of proximal and distal landing zones, the relationship of the aneurysm to the aortic arch and supra-aortic vessels, the degree of aortic tortuosity, and the size and quality of the access vessels (Fig. 22.1).

\subsubsection{Proximal Landing Zone}

The identification of a suitable proximal landing zone is one of the first factors to take in account to determine whether the endovascular technique is applicable. The proximal landing zone must be accurately measured. It is generally recommended to have at least $2 \mathrm{~cm}$ of proximal sealing to prevent distal migration. The tendency of the stent graft to follow the greater curvature and the natural tortuosity of the aorta often leads to an underestimation of the coverage length. For this reason, in cases of severe angulation, calcification, or thrombus, it may be necessary to extend the seal zone length. In approximately $20 \%$ of cases, the proximal sealing zone can only be achieved by extending across the left subclavian artery (LSA) ostium [2]. Current Society for Vascular Surgery guidelines recommend preoperative LSA revascularization in elective cases and watchful waiting in acute settings, though this remains an area of debate [3]. The presence of a left dominant vertebral artery or a left internal mammary coronary artery bypass graft is a strong indication to consider preemptive LSA revascularization. It should also be noted that the LSA can be an important input to the collateral network feeding the anterior spinal artery, and as such, if other inputs are diseased or excluded, direct revascularization should be strongly considered.

The proximity of the aneurysm to supra-aortic vessels implies to cope with arch TEVAR and the potential neurologic complications related to.

Approaches to complex TAAA with aortic arch involvement, regardless of the technique, cannot be achieved without risk. Treatment options range from open arch repair with elephant trunk or frozen elephant trunk, hybrid repair with total or partial debranching procedures, or total endovascular repair with fenestrated, branched, and scalloped devices, or parallel stent grafts (Fig. 22.2). For open aortic arch replacement, an operative mortality ranging from 0.9 to $9.3 \%$ has been reported in single series from high-volume centers [4-6].

However, according to data from the National Inpatient Sample database [7, 8] and the Medicare Provider Analysis and Review [9, 10], reflecting the real-world experience, operative mortality as high as $15 \%$ to $20 \%$ has been shown.

A variety of debranching procedures could be applied in patients with TAAA and arch involvement. In our published experience on 104 patients who underwent debranching and TEVAR for thoracic aortic disease extended to the arch, mortality was $5.8 \%$, and stroke and SCI rates were $3.8 \%$ and $2.9 \%$, respectively. Nevertheless, proximal extent of arch disease is a major determinant of outcome. Total arch debranching was confirmed in our study as an important independent predictor of 30-day mortality [11]. 

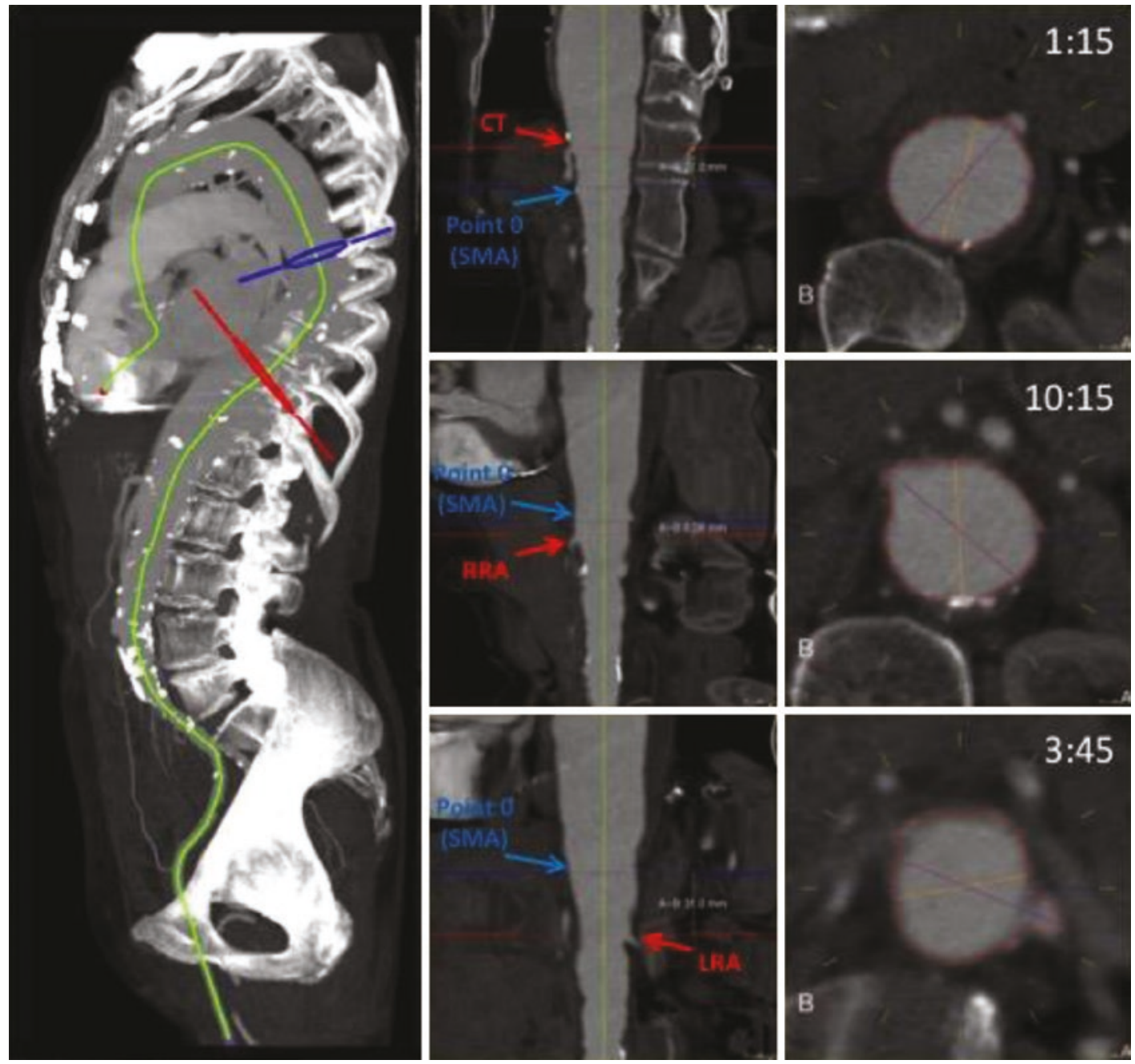

Fig. 22.1 Multiplanar reconstruction according to center lumen line for preoperative evaluation of FEVAR/BEVAR applicability ( $C T$ celiac trunk, $S M A$ superior mesenteric artery, $R R A$ right renal artery, $L R A$ left renal artery)

The recent availability of new endovascular solutions to avoid these hybrid adjuncts, limiting the invasiveness of the surgical access, may be a valid alternative to decrease intraoperative mortality.

Total endovascular therapeutic options to extend proximal landing zones in extensive TAAA range from off-the-shelf branched solutions equipped with a single branch for LSA revascularization to custom-made devices equipped with up to three fenestrations or inner branches for extension of disease to the ascending aorta (Fig. 22.3) [12, 13].

Spear and co-authors recently reported three cases of post-dissection aortic arch aneurysm treated by totally endovascular means, using a three-inner branched custom-made stent graft. The two proximal branches were antegrade for innominate and the left common carotid artery revascularization, while the third was retrograde and preloaded for LSA perfusion. Technical success was achieved in each case of this small series, and after 6 months, no patient died and no target vessel lost its patency [14].

Chimney and snorkel techniques may be applied in selected cases [15]. Such alternative procedures provide supra-aortic trunks perfusion by parallel fashion, actually representing a valid option mostly in emergent cases 

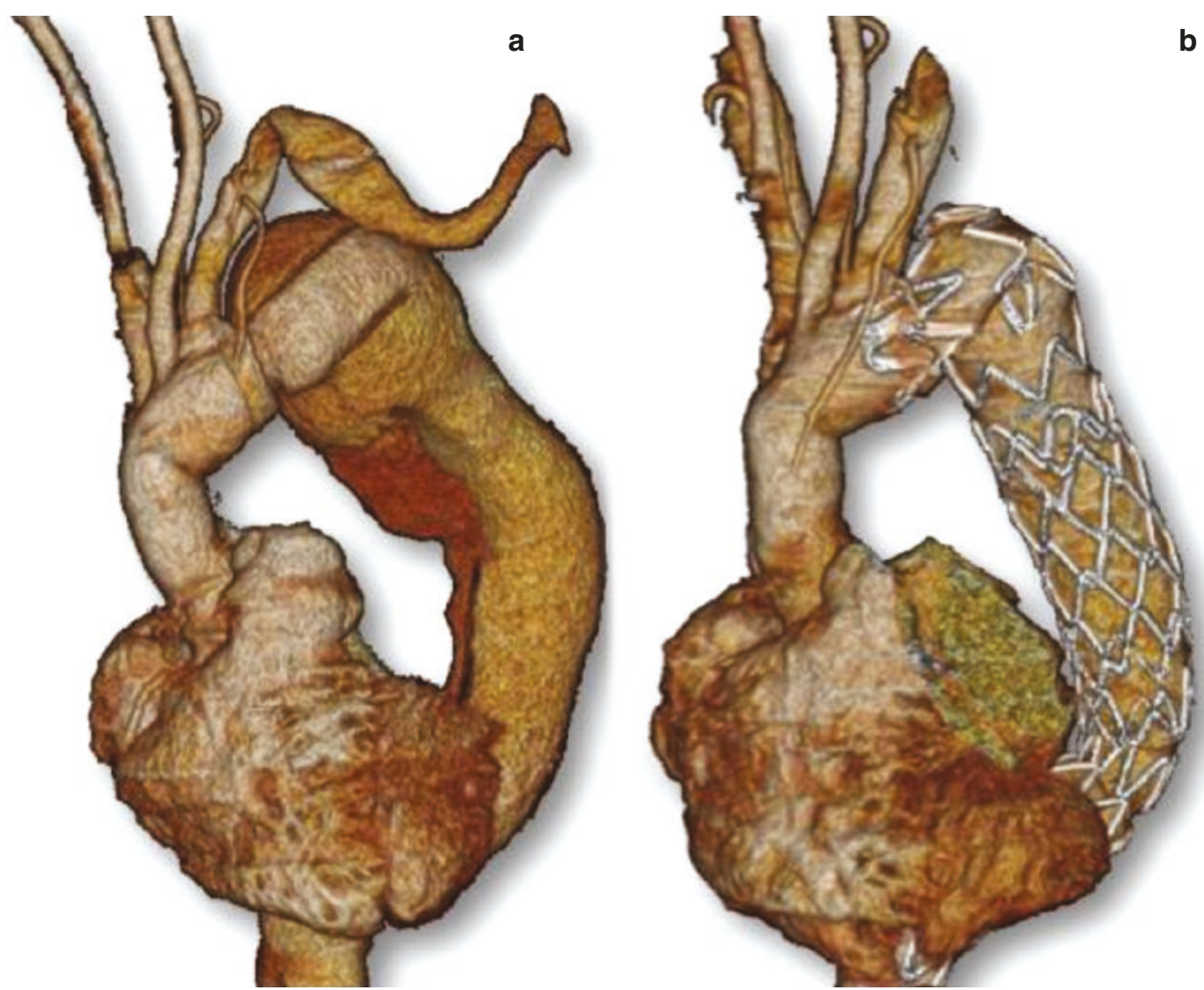

Fig. 22.2 TEVAR completion (b) after open arch repair with elephant trunk technique (a) for post-dissection TAAA with aortic arch involvement

(Fig. 22.4). Scalloped stent grafts represent another strategy in overcoming the problem of short proximal landing zone. These custommade stent grafts are equipped with a proximal scallop designed to accommodate the origin of a supra-aortic vessel. In most cases, the proximal scallop is intended for LSA preservation only, although it can be useful in particular cases where left vertebral artery arises directly from the aortic arch.

Nevertheless, the high cost and considerable time delay associated with custom-made devices have paved the way for the development of more versatile on-site modifications of stent grafts. Recently, some reports evaluated the efficacy and safety of needle, laser, or radiofrequency punctures of the aortic stent graft to create retrograde in situ fenestration for LSA or other supra-aortic trunk reperfusion [16-18]. Kasprzak et al. reported the outcome of a patient with aortic arch aneurysm treated by TEVAR and in situ fenestration for the innominate and the left common carotid artery, with in situ stented vessels still patent without any endoleak after 5 years [19].

\subsubsection{FEVAR/BEVAR Applicability}

Evaluation of anatomical applicability for endovascular TAAA repair obviously goes through the assessment of specific morphological criteria strictly related to thoracoabdominal portion of the aorta, such as aortic angulation, internal lumen diameter, visceral vessel orientation, and 


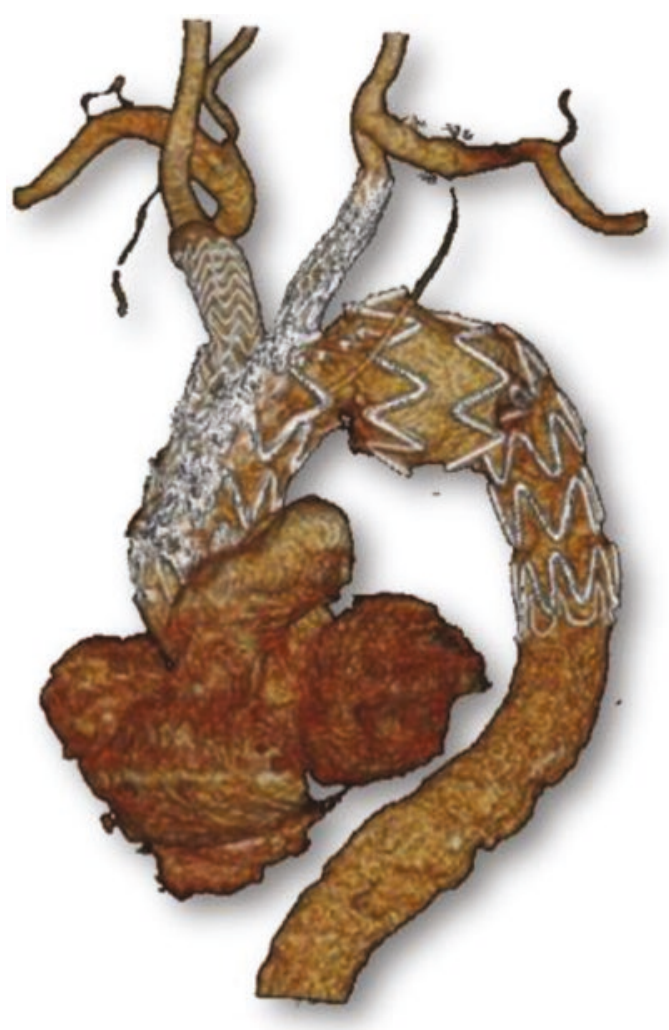

Fig. 22.3 Double inner branch device for the treatment of aortic arch aneurysm

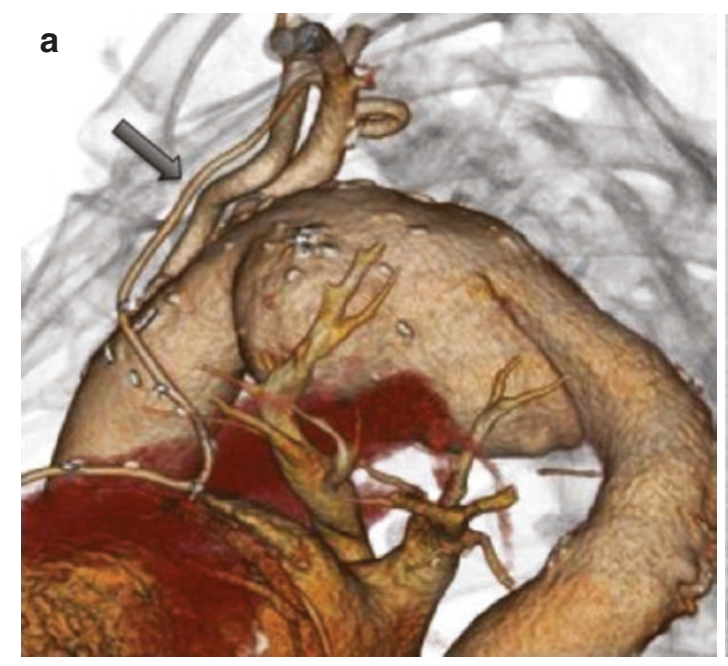

Fig. 22.4 Symptomatic distal arch aneurysm (a) in a patient with a previous coronary artery bypass graft from left internal mammary artery (arrow), treated in emergency their possible early bifurcation. These features are needed to determine absolute endovascular feasibility and, in particular, whether a solution with fenestrations, branches, or a combination of the two is the most appropriate to fit the patient's anatomy.

Two companies currently provide thoracoabdominal branched stent grafts with tailored design: Cook (Cook Medical, Brisbane, Queensland, Australia) and JOTEC (JOTEC GmbH, Hechingen, Germany). A branched stent graft with custom features, in terms of length and proximal size, aims to reduce the aortic coverage as much as possible, with important advantages in terms of SCI. By designing the side branches close to the ostium of target vessels, the length of bridging stents is significantly reduced with benefits in terms of vessel patency. However, the custom-made design has a considerable limitation due to the relatively long manufacturing time.

This excludes cases where waiting time is not allowed as in very large, symptomatic, or ruptured TAAAs, which have to be repaired through conventional surgery or other off-the-shelf techniques, such as chimney or sandwich [20].

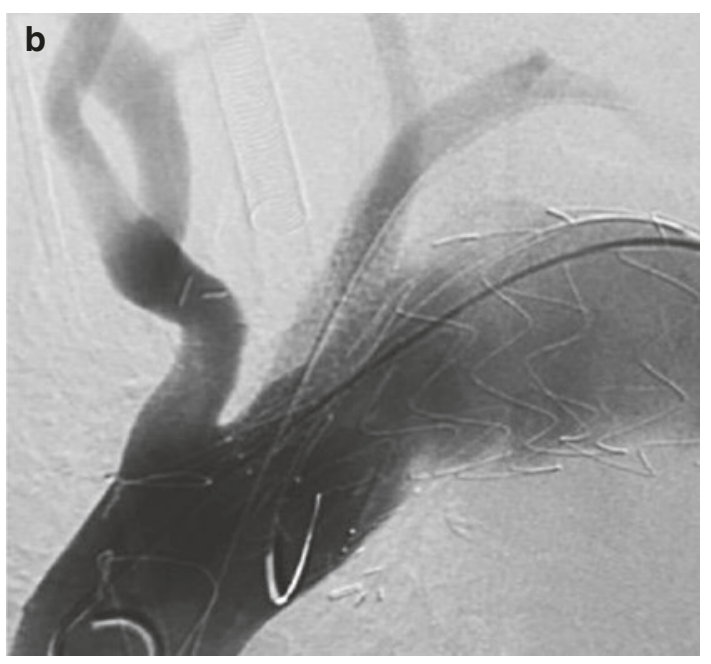

with TEVAR and double chimney (left common carotid artery and left subclavian artery) technique (b) 
To overcome these limitations, an off-theshelf multibranched device (t-Branch; Cook Medical, Bloomington, Ind, USA) has been designed. The main characteristic is the fixed position of the branches, suitable for at least $50 \%$ of TAAAs treated with a custom-made device [21]. The device consists of a proximal branched component, a distal bifurcated component, and iliac limb extensions. For extensive aortic disease, proximal thoracic aortic segments may be needed. The branches are oriented downward allowing catheterization only from axillary or brachial artery.

t-Branch use is considered feasible under the following conditions: (1) aortic diameter is $>25 \mathrm{~mm}$ at the region of the branches, (2) there are no more than four target vessels, (3) the celiac artery (CA) and superior mesenteric artery (SMA) are 6-10 $\mathrm{mm}$ in diameter, (4) the renal arteries are 4-8 $\mathrm{mm}$ in diameter, (5) all target vessels are accessible from above, (6) the distance between each branch and the corresponding arterial ostium is no longer than $50 \mathrm{~mm}$, and (7) the line between the branch and the ostium deviates by no more than $45^{\circ}$ from the main axis of the aorta.

Unused branches could be closed using an occluder plug, expanding the applicability of offthe-shelf branched devices also in cases with less than four visceral arteries.

A recently published paper on the comparison between custom-made and off-the-shelf multibranched stent grafts showed excellent technical success rates for both devices [22]. The $\mathrm{t}$-Branch is theoretically applicable in $>50 \%$ of patients with TAAAs, and this suggests that not only symptomatic or urgent cases can be treated immediately, but also elective cases are not compelled to wait for the manufacturing of the custom-made stent graft or bear the risk of aneurysm rupture during waiting. The comparable efficacy, however, does not automatically mean that the off-the-shelf stent graft should replace the custom-made device. There are some morphological features that, despite the suitability of the $\mathrm{t}$-Branch, require the performance of adjuvant procedures as additional proximal thoracic endografting or use of longer bridging stents than in custom-made device, which may lead to additional morbidity [22].

These challenges can be successfully solved by means of a custom-made device. The branches in the custom-made stent grafts are usually placed at the most optimal position above the target vessels, facilitating their catheterization. Besides, in TAAAs with a proximal landing zone $>32 \mathrm{~mm}$ in diameter, the custom-made device can be manufactured with a proximal diameter of $38 \mathrm{~mm}$ or larger. In contrast, the t-Branch device, with its fixed diameter of $34 \mathrm{~mm}$, should require a proximal extension with an additional thoracic stent graft.

Another additional disadvantage of the $\mathrm{t}$-Branch is the fixed distal diameter of $18 \mathrm{~mm}$ making the bifurcated stent graft almost always necessary.

In other words, the use of custom-made devices leads to less coverage of the aorta, facilitates the catheterization of the target vessels, and often requires fewer implanted materials.

Specific contraindications for the current models of branched devices, such as internal aortic lumen $<25 \mathrm{~mm}$, as often happens in post-dissection TAAA, and a lack of brachial or axillary access for branch catheterization, may require the use of a custom-made fenestrated stent graft, although the use of reverse cranially oriented branches, possibly customizable with inner design, may represent a valid option. These reverse branches may be useful also in cases of target vessels with severe upward orientation (Fig. 22.5).

The Gore TAMBE off-the-shelf branched endograft (W.L. Gore \& Associates, Flagstaff, Az, USA) features four inner branches, two antegrade and two retrograde. This characteristic leaves more room between the stent graft and the ostia of target vessels, possibly making catheterization maneuvers easier and expanding the applicability of branched stent grafts for TAAA with small internal lumen. The staged deployment of this new design branched stent graft, consisting first in the opening of the proximal and distal endpoints and then of the visceral docking area, may allow a full catheterization of the four target vessels before the complete expansion of the branched stent graft. The four inner branches, 


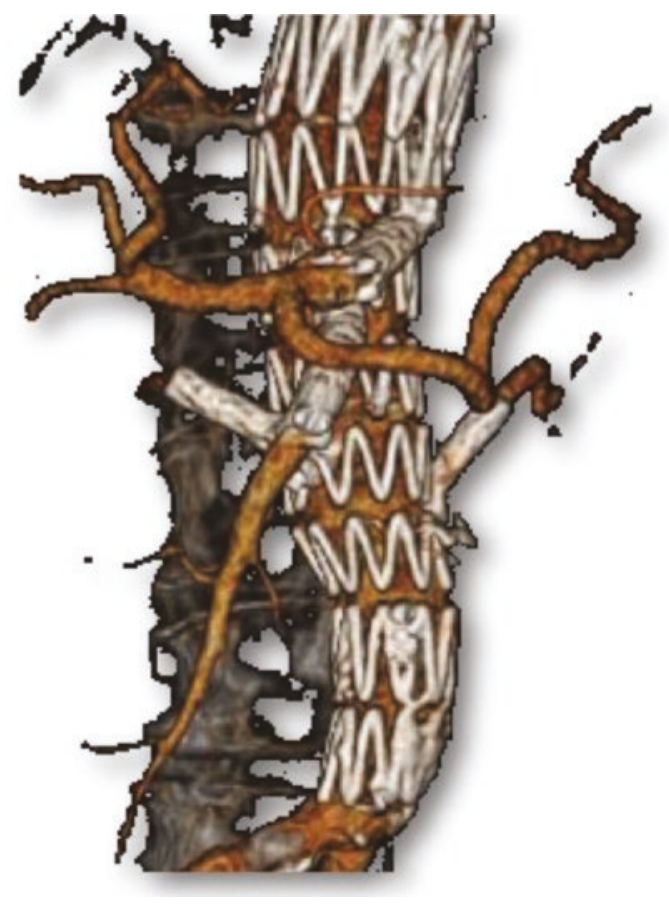

Fig. 22.5 Custom-made stent graft for type III TAAA with two branches for celiac artery and superior mesenteric artery and two fenestrations for cranially oriented renal arteries

two antegrade for CA and SMA and two retrograde for renal arteries, are pre-cannulated with two through-and-through catheters, allowing the catheterization of the four target vessels (CA and SMA from above and renals from below) with the branched stent graft still partially constricted.

The graft has only been used in very few cases, and all these features need to be tested in a reasonable number of patients.

A fenestrated stent graft is custom-made to fit patient anatomy in terms of length, number, and position of fenestrations. In cases of extensive aortic disease, proximal tubular components may be needed. There are two types of fenestrations that can be manufactured in the fenestrated component, including small and large fenestrations. Small fenestrations have dimensions of $6 \times 6 \mathrm{~mm}$ or $6 \times 8 \mathrm{~mm}$. Large fenestrations measure $8-12 \mathrm{~mm}$ in diameter. A nitinol ring reinforces all fenestrations.

Contraindications to the use of fenestrated devices often relate to the angulation of visceral aorta and the possible severe internal lumen narrowing, as sometimes happens at renal artery removal. The choice of a fenestrated stent graft in these conditions may result in a twisting during the deployment with unpredictable misalignment of the fenestrations and dramatic consequences on target vessel reperfusion. The presence of a previous surgical infrarenal graft may limit the ability to adjust the stent graft position during and after the deployment. For this reason, single or double diameter-reducing ties are usually placed along the posterior aspect of fenestrated stent grafts in order to limit the initial expansion of the graft after sheath withdrawal, thereby reducing the device profile and increasing longitudinal and axial maneuverability.

\subsubsection{Access Vessels}

In the planning path of TAAA endovascular repair, once the proximal landing zone has been identified and branched/fenestrated feasibility established, ascertaining suitable access vessels is the final hurdle for endovascular applicability. Whereas hydrophilic sheaths have reduced trauma to iliofemoral axes, the inadequate size of the access vessels, especially in women or calcified and tortuous arteries, may represent a contraindication for TAAA endovascular treatment. The passage of large-caliber devices precludes safe transfemoral TEVAR in up to $30 \%$ of patients [23]. In some cases, an iliac or aortic conduit may be needed, increasing the risk of hemorrhage and hemodynamic instability and possibly leading to increased mortality and morbidity, including SCI. Published series of TEVAR report a 9-22\% incidence of access vessel complications, contributing to perioperative morbidity in patients who are often elderly and fragile [24-26].

Arnaoutakis et al. reported the outcomes of TEVAR procedures from the American College of Surgeons National Surgical Quality Improvement Program database. A total of 649 patients were evaluated in this report. The 279 women who were included were more likely to require iliac artery access when compared with men (18\% vs 
$7 \% ; P<0.001)$, and this alternative approach was identified as an independent predictor of 30-day mortality (relative risk, 4.42; 95\% confidence interval, 2.07-9.44; $P<0.001)$ [27]. In a series of 164 patients, as reported by Lee et al., an iliac conduit resulted in a 2.6-fold increase in blood loss, $82 \%$ longer procedure time, 1.5 additional hospitalization days, and a 1.8-fold higher rate of perioperative complications [28]. Current limitations in TEVAR have recently been addressed with new design, low-profile thoracic stent grafts. Melissano and co-authors reported their initial experience with the Cook Alpha low-profile thoracic stent graft (Cook Medical, Bloomington, Ind, USA) in 42 patients with descending thoracic aortic disease. In this series, including 11 patients with concomitant aortoiliac occlusive disease (TransAtlantic Inter-Society Consensus type B and C), technical success in terms of safe and effective deployment of the thoracic stent graft was $100 \%$, and no access-related complications were recorded. Furthermore, in patients with associated aortoiliac disease, no adjunctive procedures for device insertion, except for predilation with balloon angioplasty in two cases, were required [29].

Although the graft material alterations employed to achieve lower profile could theoretically lead to limited durability, mid-term results seem to be promising. In a series of 70 consecutive patients treated with the Zenith Alpha stent graft published by Torsello and co-workers, just three cases of type Ia endoleak (4.3\%), two cases of type Ib endoleak (2.9\%), and one case of aneurysm sac enlargement (1.4\%) were recorded at a follow-up period of $22.3 \pm 15.9$ months. Re-intervention was necessary in only one case $(1.4 \%)$ and no stent fracture was reported in this study group [30].

Low-profile technology was soon applied to multibranched thoracoabdominal devices (Fig. 22.6). Ramanan et al. published a comparative study on 134 pararenal or TAAA treated with standard (97) and low-profile (37) multibranched stent graft. Main outcomes were similar between the two groups. However, a combined outcome of conduit use or access artery injury occurred at a lower rate in the low-profile than in the standard-profile group (16\% vs 36\%; $P=0.03$ ). Furthermore, women experienced significantly higher rates of conduit use and access artery injury than men after repair with standard-profile (64\% vs $26 \%$, respectively; $P=0.001$ ) but similar rates after repair with low-profile branched stent graft (25\% vs $14 \%$, respectively; $P=0.45$ ) [31].

The possibility of today applying total endovascular solutions for TAAA with devices loaded onto introducing sheath of $18-20 \mathrm{~F}$ in outer diameters makes the percutaneous approach particularly appealing. Although the use of percutaneous technique for TAAA endovascular repair is still debated, this approach is theoretically associated with lower blood loss from the groins, contributing to a more stable hemodynamic status of the patient during the entire procedure. Furthermore, sheath withdrawal from the iliac arteries immediately after deploying the aortic device and before bridging the visceral vessels, with consequent early restoration of blood flow to the pelvis and lower limbs, has proven to be effective in terms of SCI prevention [32].

de Souza et al. reported a $95 \%$ of technical success for total percutaneous endovascular aortic repair for thoracic, fenestrated, and branched stent grafts. In this series of 170 femoral arteries approached with $\geq 20$-F-diameter sheaths, accessrelated complications occurred in $5 \%$ of cases within 30 days, and no late access-related events were reported [33].

\subsection{Outcome Evaluation}

\subsubsection{Open Repair}

Open repair of TAAAs is still associated with high rates of postoperative mortality and morbidity. Studies on national data report mortality rates as high as $20 \%$ after open surgical repair $[8,34]$, whereas large single-institution reviews report mortality rates as low as 5-8\% [35-37]. Although increasing surgical and hospital volume have been associated with improved outcomes after open surgical repair, postoperative complications remain common and include 

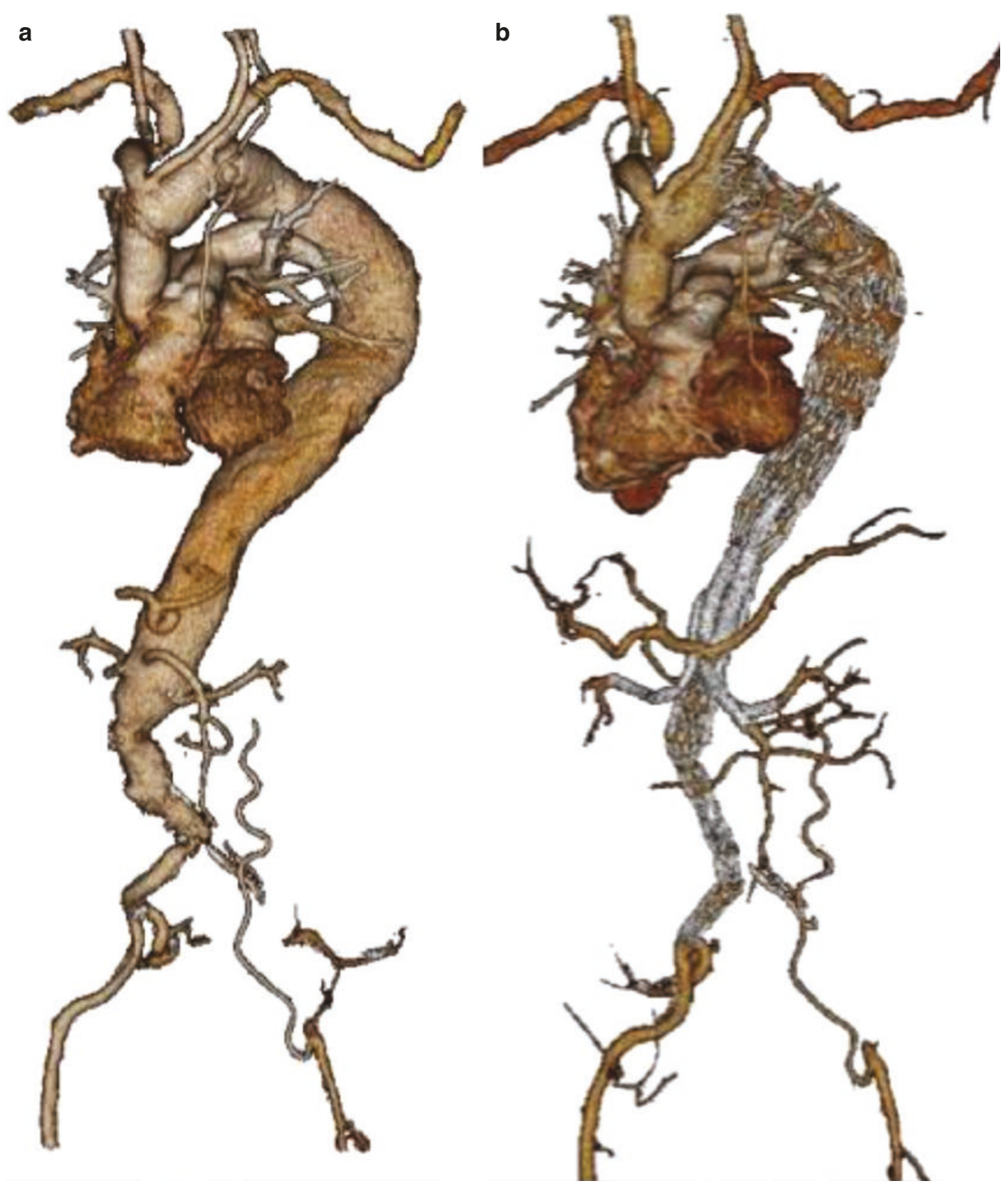

Fig. 22.6 Type II TAAA (a) treated with low-profile multibranched stent graft and aorto-uni-iliac distal extension for concomitant left iliac axis chronic occlusion (b)

pulmonary, cardiac, and renal complications, as well as spinal cord ischemia (SCI). Perioperative management of patients undergoing TAAA surgery has evolved substantially during the past two decades, resulting in improved early survival and decreased morbidity. The fact that the results are influenced by the caseload was already evident from historical reports like that by Svensson et al. on Houston's experience. In this series of 1509 patients undergoing TAAA open repair from 1960 to 1991, the reported 30-day mortality and SCI rates were $8 \%$ and $16 \%$, respectively 
[37]. As there is no support from current strategies on organ protection, these values must be considered more than acceptable. Over the last few years, a variety of operative approaches and protective adjuncts have been introduced to minimize organ dysfunction and, in particular, SCI. Conrad et al. reported on 445 consecutive TAAA open repairs with 30-day mortality of $8.2 \%$, with a SCI of any severity of $13.2 \%$, of which $9.5 \%$ sustained permanent paraplegia. Predictors of SCI included extent I/II aneurysms, epidural cooling, urgent/emergent operation, intraoperative hypotension, total aortic crossclamp time, and postoperative pulmonary complications. Late survival rates in this series were $54.4 \%, 28.7 \%$, and $20.5 \%$ at 5,10 , and 15 years, respectively [35]. Coselli et al. in one of the largest published series reported 30-day mortality, $\mathrm{SCI}$, and renal failure requiring hemodialysis of $5 \%, 3.8 \%$, and $5.6 \%$, respectively. Intraprocedural adjuncts used were segmental arteries reattachment in $61.3 \%$ of patients, cerebral spinal fluid drainage in $26.9 \%$, and left heart circulatory support in $39.8 \%$ [36].

Data from the National Surgical Quality Improvement Program on 450 patients undergoing TAAA open repair showed a 30-day mortality and morbidity rate of $10 \%$ and $51.6 \%$, respectively. Pulmonary complications were the most common, occurring in $45.1 \%$ of patients. Cardiac complications were observed in $6.4 \%$ of cases. Renal failure requiring hemodialysis occurred in $10.7 \%$ of patients, while neurologic complications were more common in emergent than in elective cases $(12.5 \%$ vs $1.4 \% ; p=0.003)$ [38]. Many advances have been done also in techniques for renal protection during TAAA open repair. Tshomba et al., in a comparative study on 111 TAAA patients propensity matched according to the type of intraoperative renal perfusion, found that the use of Custodiol provided improved perioperative renal function compared with lactated Ringer's solution [39].

Despite these improvements, the management of patients affected by TAAA remains challenging, and subjects with comorbidities and extensive aneurysms remain at high risk for postoperative complications.

\subsubsection{Endovascular Repair}

\subsubsection{FEVAR and BEVAR}

Since the first case of branched stent graft for TAAA reported by Chuter et al. in 2001 [40], following the progressive diffusion of this technology worldwide, several case series have been published over the last few years with satisfactory results in terms of short-term mortality and morbidity. The less-invasive nature of the endovascular approach is intended to diminish the magnitude of the repair by limiting groin incisions, avoiding aortic cross-clamping, and minimizing perioperative end-organ ischemia, blood loss, fluid shifts, and injury to the cardiac and respiratory systems. However, there is no evidence of decreased incidence of SCI. Furthermore, late complications, such as endoleaks, stent fractures, or migration, unknown for open repair, can affect the long-term durability of endovascular treatment and should be balanced with the late complications and re-intervention occurring after open repair [35].

One of the largest experiences with endovascular treatment of TAAAs has been provided by the Cleveland Clinic on a series of 406 patients enrolled in a prospective study with 2-year follow-up data. Perioperative mortality was $12.5 \%$ for type I, $5.2 \%$ for type II or III, and $2.3 \%$ for type IV TAAA according to Crawford's classification. Symptoms of SCI were noted in $4.3 \%$ of the overall endovascular group (also including 227 AAAs in addition to the 406 TAAAs). Overall survival at 2 years was $70 \%$ for type I, $74 \%$ for types II and III, and 82\% for type IV TAAA [41].

More recently, Eagleton and co-authors published a series of 354 high-risk patients, again from Cleveland Clinic, treated with FEVAR or BEVAR for type II and III TAAA. Thirtyday mortality occurred in 17 patients (4.8\%), permanent signs of SCI were observed in 14 patients (4\%), and renal failure requiring hemodialysis occurred in 10 (2.8\%). Eighty endoleak repairs were performed in 67 patients from this series, including 55 branch-related endoleaks. At 36 months, freedom from aneurysm-related death was $91 \%$ (95\% CI, 0.88-0.95), and freedom from all-cause mortality was $57 \%$ (95\% CI, 
0.50-0.63). The authors found that extent type II TAAA $(P<0.01)$, age $(P<0.01)$, and chronic obstructive pulmonary disease $(P<0.05)$ negatively affected survival [42].

There is evidence that continuous refinements with the endovascular technique based on accumulated experience have decreased risk of SCI after FEVAR and BEVAR. The French experience of 204 endovascular TAAA repairs using custom-made devices manufactured with branches and fenestrations from 2004 to 2013 reported favorable outcomes after the implementation of an innovative protocol since 2010 that included optimization of the device implantation technique (early restoration of arterial flow to the pelvis and lower limbs, staged approach) and the systematic application of the proactive spinal cord protective protocol. The authors found that the introduction of the new protocol allowed a significant decrease of SCI risk from $14.0 \%$ to $1.2 \%(p<0.01)$ and a benefit in halving the 30 -day mortality from $11.6 \%$ to $5.6 \%$, even though it was not statistically relevant $(p=0.09)$. The benefit was particularly evident in reducing the rates of SCI after type I to type III TAAA ER (excluding type IV): $25.0 \%$ (6/24 patients) before the protocol and $2.1 \%$ (2/95 patients) after the protocol $(p<0.01)$ [32].

Data supporting that endovascular repair of TAAA has today reached a state of maturity with early results at least comparable with open surgery are reported also from Mayo Clinic. Oderich and his group reported a 30-day mortality of $4.3 \%$ for a group of 185 patients with TAAA, including mortality of $1.8 \%$ for extent IV and $8.2 \%$ for extent I to III $(P=0.03)$. The authors underlined that overall mortality decreased in the second half of their clinical experience from $7.5 \%$ to $1.2 \%$. SCI occurred in two patients $(1.8 \%)$ with type IV TAAA and four $(3.2 \%)$ with extent I-III. At 5 years, primary patency of stented target vessels was $93 \%$ [43].

Similar results also came out of high-volume European centers [44].

Verhoeven et al. described the outcomes of 166 patients with TAAA treated with fenestrated or branched devices, reporting a perioperative mortality of $7.8 \%$ and SCI of $9 \%$ (of which $1.2 \%$ leading to permanent paraplegia) and an estimated survival at 1,2 , and 5 years of $83 \%, 78 \%$, and $66 \%$, respectively [45].

The same group showed one of the largest experiences with the use of fenestrated or branched devices in post-dissection TAAA. Oikonomou and colleagues described the outcome of 31 patients treated with FEVAR or BEVAR for aneurysmal degeneration of chronic dissection. Technical success was $93.5 \%$ and 30 -day mortality $9.6 \%$. No case of permanent paraplegia was reported, and the seven late deaths recorded in a mean follow-up of 17 months were all unrelated to aneurysms (Fig. 22.7) [46].

Some centers have published information about the durability of complex endovascular repair, especially with regard to re-intervention and branch-related events.

Reilly et al. described their experience with 81 patients who underwent repair with branched stent graft [47]. The overall mortality in this series was $6.2 \%$ over a mean follow-up of 21 months. The rate of SCI was $3.7 \%$; however, paraparesis was observed in $20 \%$ of cases.

Intraoperative injury of target vessels occurred in $4.6 \%$ of branches, the majority of which were treated immediately, with only one requiring occlusion to treat bleeding. Over the course of follow-up, stenosis developed in five patients (renal branches in four and the SMA branch in one). Of the 81 patients, 4 demonstrated aneurysm enlargement. Two of these patients required intervention and extension of the stent graft. An additional ten patients required intervention for persistent endoleaks. Only two endoleaks developed after the 1-month CTA. In total, 32 patients underwent 42 re-interventions over 21 months.

In another publication focusing on the durability of FEVAR and BEVAR, the authors analyzed a cohort of 650 patients undergoing endovascular repair with combined branch and fenestrated devices [48]. The mean follow-up was 3 years ( standard deviation $=2.3$ ), and during the 9 years of maximum follow-up, secondary procedures were required for $6 \%$ of right renal artery, $5 \%$ of left renal artery, $0.6 \%$ of CA, and $4 \%$ of SMA branches. The need for re-intervention was bimodal but on average appeared at 237 days 

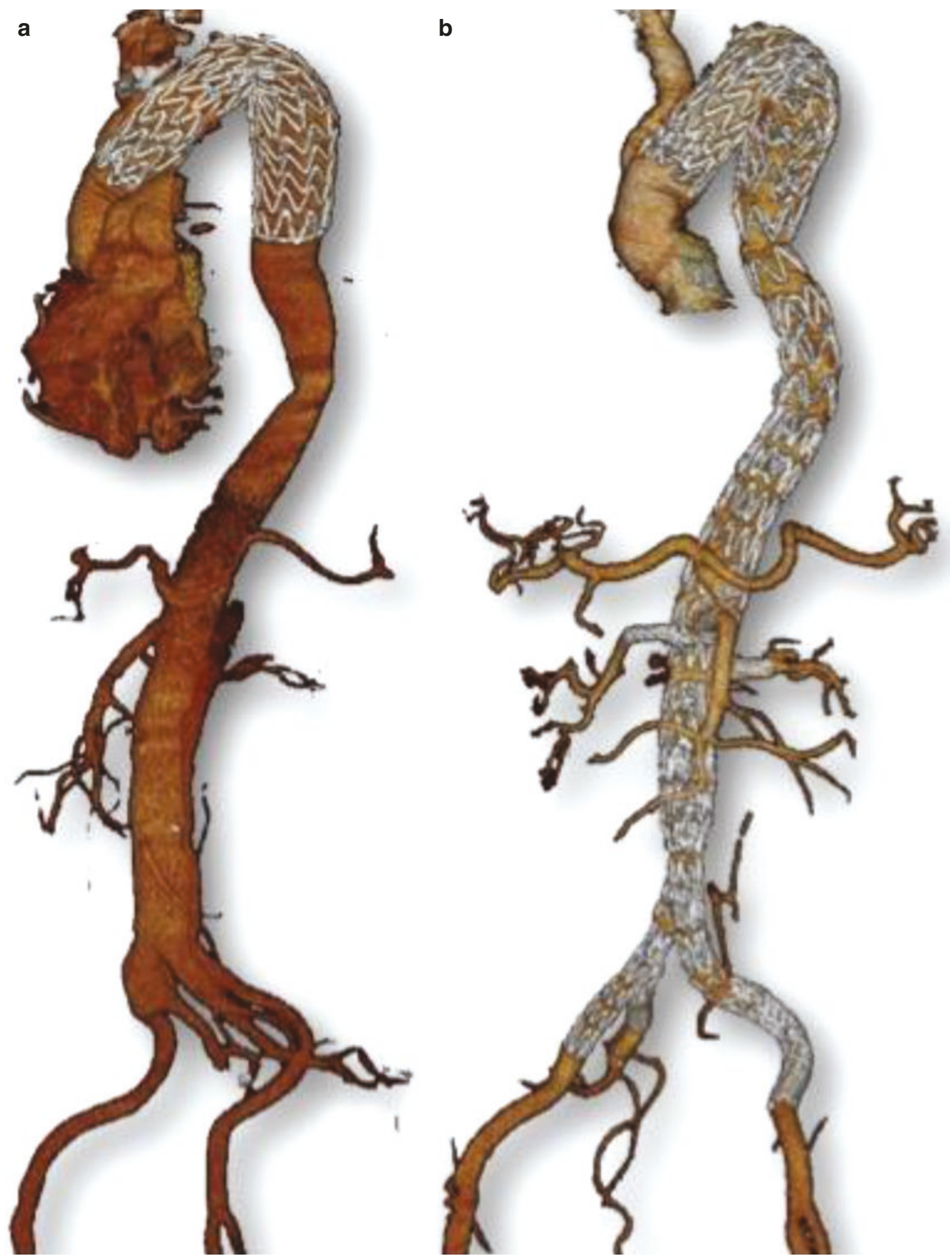

Fig. 22.7 Staged repair with custom-made fenestrated stent graft and iliac branch device (b) for post-dissection type II TAAA (a) 
after index repair. Death from branch stent complications occurred in three patients, all involving the SMA.

The authors recommend that a composite outcome of branch instability, which includes branch occlusion, device migration affecting a branch, branch-related growth, and the need for any secondary intervention in a branch, is to the best metric to assess the durability of repair.

More recently, again from Cleveland Clinic, long-term outcomes of 1091 FEVAR/BEVAR patients were published. Non-risk-adjusted survival at 30 days, 1 year, and 5 years was $96.8 \%$, $85.1 \%$, and $46 \%$, respectively. It is of interest that freedom from aneurysm-related death was $96.8 \%$, $95.2 \%$, and $94.0 \%$ at 30 days, 1 , and 5 years, respectively. The authors also found that older age, heart failure, chronic obstructive pulmonary disease, renal disease, anemia, and coagulation disorders were risk factors for late mortality [49].

\subsubsection{Parallel Endografting}

Parallel stent grafts, including the chimney, snorkel, and sandwich techniques, are a proposed strategy for increasing the applicability of endovascular repair to aneurysms involving branch vessels. One major disadvantage of this strategy is the imperfect nature of the seal, which is inherent to having multiple side-by-side stent grafts. In fact, the major objection against a chimney-based procedure is the gutter (the channel between the parallel grafts), which can prevent immediate sealing and exclusion of the aneurysm. The sandwich technique was introduced to offer an endovascular off-the-shelf solution to patients with TAAA as an alternative to a custom-made device $[50,51]$. Initially described in the treatment of aortoiliac disease, it combines the principles of a chimney graft with aneurysm exclusion by using several aortic stent grafts overlapped [52]. A chimney graft runs parallel to an aortic stent graft into the visceral or renal vessel, and there is a direct contact between the chimney graft and the aortic wall. The limitation in length of the chimney grafts does not permit endovascular exclusion of TAAA. In a sandwich procedure, the parallel stents run into the space created by two aortic stent grafts [53].
The first report about parallel endografting, published by Lobato et al., included patients with complex aortic aneurysm that did not fulfill the requirements for standard endovascular repair. They included patients with aortoiliac, TAAA, and aortic arch disease [50].

Schwierz et al., focusing on patients with TAAA, reported their experience on 104 chimney grafts implanted with sandwich technique in 32 patients with TAAA [54]. Indication for the procedure in $43 \%$ was an acute onset of symptoms, including two patients with a rupture and a retroperitoneal hematoma. Two patients died postoperatively because of the operation. Major adverse events were recorded in five patients, including one patient with persistent paraplegia and two with permanent renal failure requiring dialysis. The incidence of chimney graft occlusion was higher in patients with three or four parallel grafts than in those with two chimney grafts only. Patients with chronic dissections had a 12-times higher incidence of chimney graft occlusion than aneurysm patients. Type I or III endoleak was more common in the group with three or four parallel grafts [54]. Sandwich technique requires advanced endovascular techniques and imaging comparable to the deployment of any fenestrated or branched device. The presence and the size of the gutters between the chimney stents and the aortic stent grafts have always been an issue since the first publication of this technique. In a recent report, de Bruin et al. showed experimentally that balloon-expanding stents had larger gutters compared with self-expanding branches [55], and the compression of the main grafts increased with the size of the chimney grafts. Promising results were obtained in an experimental study with endostaples that were used to reduce the size of the gutters [56].

If the sandwich technique requires advanced endovascular techniques and imaging comparable to FEVAR and BEVAR on the one hand, it exposes patients to higher risk of endoleak on the other. For this reason, in our opinion, the use of this technique should not be routine, but reserved to those cases in which fenestrated or branched solutions are not available or definitely not applicable. 


\subsubsection{Open Versus Endovascular Repair}

The decision about which therapy is appropriate for a particular patient is an evolving aspect of care of these complicated patients. Only three industry-sponsored trials comparing surgical and endovascular repair of thoracic aortic aneurysms were published [57-60], and no randomized, controlled prospective trial comparing open and endovascular TAAA repair exists. In addition, comparative studies including only pure TAAA are very few.

The largest series comparing open versus endovascular treatment for TAAA was published by Cleveland's group in 2008 [61]. A total of 372 patients were included in the OR group and 352 in the ER group. Patients treated with ER were on average 8.6 years older $(71.3 \pm 12$ versus $62.7 \pm 13$ years, $p<0.001)$ than those treated with OR. In this study the 30-day mortality rate was $8.3 \%$ in OR and $5.7 \%$ in ER patients $(p=0.2)$ and 1 -year mortality $15.9 \%$ and $15.6 \%$, respectively $(p=0.9)$. SCI was detected in $15 \mathrm{ER}$ patients $(4.3 \%)$ and 28 OR patients $(7.5 \%$; logrank $p=0.08)$, but at univariable and multivariable analysis, and after propensity score adjustment, the repair technique used was not associated with the presentation of SCI. In contrast, the extent of aneurysm repair was strongly associated with the development of SCI regardless of statistical adjustments. In both these groups, the occurrence of SCI was highest for type II aneurysms, followed by types I, III, and then IV. The severity of the SCI and the potential for recovery did not differ between treatment modalities. In their experience, the authors noted that SCI was more commonly observed immediately after OR (29\% versus $13 \%$ ) and in a delayed presentation (up to 6 days) after ER. A prior distal aortic operation results at the univariable analysis as a risk factor for the development of SCI ( $p=0.004)$ in ER patients.

We recently published the results of a comparative analysis of outcome after OR and ER for patients with TAAA referred to three vascular centers from 2007 to 2014 [62]. Patients in the OR group were matched to patients in the
ER group on the basis of propensity score. The model included all the baseline characteristics that were reliably measured and were clinically relevant in driving the endovascular or open treatment assignment. The aim of this method was to generate two groups of patients with correction of all differences in baseline characteristics. On the basis of our results, the 65 patients included in the ER and OR groups had similar rates of perioperative mortality $(7.7 \%$ vs $6.2 \%)$ and renal complications $(9.2 \%$ vs $12.3 \%)$. The incidence of SCI was $12.3 \%$ in the ER group and $20 \%$ in the OR group, a difference that was not statistically significant and disappeared when only rates of permanent paraplegia were considered (9.2\% vs $10.8 \%$ in ER and OR, respectively). However, OR exposed the patients to a major overall morbidity burden as shown by the two times higher rate of a composite endpoint (including any SCI, renal and respiratory insufficiency) compared with the ER group: $36.9 \%$ vs $18.5 \%$ after ER and OR, respectively. Furthermore, as expected, the 30-day rate of respiratory insufficiency was significantly lower in ER ( $0 \%$ vs $12.3 \% ; p=0.006$ ) [62]. These findings may help in selecting the open versus endovascular treatment for TAAA tailored to the single patient, specifically with regard to the effect of OR on respiratory function.

In our comparative study, at a median followup of 21.6 months, all-cause survival at 24 and 42 months was $82.8 \%$ in ER and $84.9 \%$ in OR $(p=0.9)$. No aorta-related late deaths occurred after ER, whereas one patient in the OR group died of sepsis secondary to an aortobronchial fistula. Freedom from re-intervention rates was $91.0 \%$ in ER vs $89.7 \%$ in OR at 24 months and $80.0 \%$ in ER vs $79.9 \%$ in OR $(p=0.3)$ at 42 months. Our data on re-interventions are partially in disagreement with previous studies showing uncertainty about the durability of ER and reporting a need for re-intervention as high as $26 \%$ at a mean follow-up of 2 years after the endovascular procedure for complex aortic disease even though most re-interventions were required for type III endoleak, similar to other reports [63-65]. This divergence may in part be explained by the inclusion, in the previous study, of both juxta-renal and thoracoabdominal 
aneurysms, causing differences in patient and anatomy selection.

The findings of our study should be interpreted with caution; propensity analysis did not allow a totally balanced comparison as by a randomized trial and resulted in a well-matched but small subset of TAAA patients. This may explain different findings reported by other studies. In a combined French and American institutional experience, the comparison of 147 open and 42 fenestrated repairs for juxta-renal aneurysms with propensity score matching showed unsatisfying results for the endovascular approach. Mortality $(9.2 \%$ vs $2 \% ; p=0.05)$ and procedural complications $(24 \%$ vs $7 \% ; p<0.01)$ were significantly higher in the endovascular than in the open repairs. Despite the fact that the comparison was focused on patients with juxta-renal aneurysms and TAAAs were excluded, reflecting less challenging diseases to be treated, the endovascular approach was independently associated with fivefold increased 30-day mortality risk (odds ratio, $5.1 ; 95 \% \mathrm{CI}, 1.1-24 ; p=0.04$ ). These worse results with fenestrated devices may likely be explained by the older timing of the study ranging from 2001, reflecting the effect of developing training and techniques [66].

To encompass the variability of TAAA patients in the real world, we suggest that open and endovascular repair should not be offered at random, but it is more likely that the choice should be individualized to achieve the best outcomes in different patients. This message may be important also because fenestrated and branched stent grafts, although initially designed to treat only high-risk patients with complex aortic aneurysms, are today increasingly applied in average-risk patients as a less invasive alternative to open repair.

In conclusion, it is reasonable to state that TAAA repair is today performed with respectable results mainly in high-volume centers, regardless of the technique. Even though longer assessment is required to confirm the stability and durability of fenestrated and branched stent grafts, the reduced early morbidity associated with ER may have an impact on decision-making for older and high-risk as well as for young and lower-risk patients.

\subsection{Conclusion}

The myth of an entirely endovascular world for thoracoabdominal aortic disease should today be considered reality. The unbridled development of FEVAR and BEVAR and the incessant technique refinement, with combination of custom-made and off-the-shelf solutions, made endovascular repair widely applicable, with results at least comparable to a well-defined treatment like open surgery.

However, re-intervention rate after endovascular repair is not negligible. The issue of increased cost (initial cost of the stent grafts, multiple CT scans in follow-up, secondary procedures) will also need to be figured into the equation in considering which therapy is the most appropriate for a particular patient. Furthermore, late survival is poor but is caused mostly by nonaneurysmrelated deaths, underlying the short life expectancy of a population of elderly patients with poor preoperative status.

With this in mind, it is today more clear that FEVAR and BEVAR should be further evaluated in low- to average-risk patients who will likely experience better long-term survival.

\section{References}

1. Mastracci TM, Greenberg RK. Complex aortic disease: changes in perception, evaluation and management. J Vasc Surg. 2008;48(Suppl 6):17S-23S. discussion 23S.

2. Cheng D, Martin J, Shennib H, et al. Endovascular aortic repair versus open surgical repair for descending thoracic aortic disease a systematic review and meta-analysis of comparative studies. J Am Coll Cardiol. 2010;55(10):986-1001.

3. Matsumura JS, Rizvi AZ, Society for Vascular Surgery. Left subclavian artery revascularization: Society for Vascular Surgery Practice Guidelines. J Vasc Surg. 2010;52(Suppl 4):65S-70S.

4. Patel HJ, Nguyen C, Diener AC, Passow MC, Salata D, Deeb GM. Open arch reconstruction in the endovascular era: analysis of 721 patients over 17 years. J Thorac Cardiovasc Surg. 2011;141:1417-23.

5. Iba Y, Minatoya K, Matsuda H, Sasaki H, Tanaka H, Kobayashi J, et al. Contemporary open aortic arch repair with selective cerebral perfusion in the era of endovascular aortic repair. J Thorac Cardiovasc Surg. 2013;145(3 Suppl):S72-7. 
6. Thomas M, Li Z, Cook DJ, Greason KL, Sundt TM. Contemporary results of open aortic arch surgery. J Thorac Cardiovasc Surg. 2012;144:838-44.

7. Sachs T, Pomposelli F, Hagberg R, Hamdan A, Wyers $\mathrm{M}$, Giles $\mathrm{K}$, et al. Open and endovascular repair of type B aortic dissection in the Nationwide Inpatient Sample. J Vasc Surg. 2010;52:860-6.

8. Cowan JA, Dimick JB, Henke PK, Huber TS, Stanley JC, Upchurch GR. Surgical treatment of intact thoracoabdominal aortic aneurysms in the United States: hospital and surgeon volume-related outcomes. J Vasc Surg. 2003;37:1169-74.

9. Patel VI, Mukhopadhyay S, Ergul E, Aranson N, Conrad MF, Lamuraglia GM, et al. Impact of hospital volume and type on outcomes of open and endovascular repair of descending thoracic aneurysms in the United States Medicare population. J Vasc Surg. 2013;58:346-54.

10. Chikwe J, Cavallaro P, Itagaki S, Seigerman M, Diluozzo G, Adams DH. National outcomes in acute aortic dissection: influence of surgeon and institutional volume on operative mortality. Ann Thorac Surg. 2013;95:1563-9.

11. De Rango P, Cao P, Ferrer C, Simonte G, Coscarella C, Cieri E, et al. Aortic arch debranching and thoracic endovascular repair. J Vasc Surg. 2014;59: 107-14.

12. Haulon S, Greenberg RK, Spear R, Eagleton M, Abraham C, Lioupis C, et al. Global experience with an inner branched arch endograft. J Thorac Cardiovasc Surg. 2014;148:1709-16.

13. Spear R, Haulon S, Ohki T, Tsilimparis N, Kanaoka Y, Milne CP, Debus S, Takizawa R, Kölbel T. Subsequent results for arch aneurysm repair with inner branched endografts. Eur J Vasc Endovasc Surg. 2016;51:380-5.

14. Spear R, Clough RE, Fabre D, Roeder B, Hertault A, Martin Gonzalez T, Azzaoui R, Sobocinski J, Haulon $\mathrm{S}$. Total endovascular treatment of aortic arch disease using an arch endograft with 3 inner branches. J Endovasc Ther. 2017;24(4):534-8.

15. Moulakakis KG, Mylonas SN, Dalainas I, Sfyroeras GS, Markatis F, Kotsis T, et al. The chimney-graft technique for preserving supra-aortic branches: a review. Ann Cardiothorac Surg. 2013;2(3):339-46.

16. McWilliams RG, Murphy M, Hartley D, et al. In situ stent graft fenestration to preserve the left subclavian artery. J Endovasc Ther. 2004;11:170-4.

17. Manning BJ, Ivancev K, Harris PL. In situ fenestration in the aortic arch. J Vasc Surg. 2010;52:491-4.

18. Redlinger RE, Ahanchi SS, Panneton JM. In situ laser fenestration during emergent thoracic endovascular aortic repair is an effective method for left subclavian artery revascularization. J Vasc Surg. 2013;58:1171-7.

19. Kasprzak PM, Kobuch R, Schmid C, Kopp R. Longterm durability of aortic arch in situ stent graft fenestration requiring lifelong surveillance. J Vasc Surg. 2017;65(2):538-41.

20. Bisdas T, Donas KP, Bosiers M, Torsello G, Austermann M. One-year follow-up after total endovascular repair of a contained-ruptured thoracoabdominal aortic aneurysm with the sandwich technique. J Vasc Surg. 2013;58:673-8.

21. Bisdas T, Donas KP, Bosiers M, Torsello G, Austermann M. Anatomical suitability of the t-branch stent graft in patients with thoracoabdominal aortic aneurysms treated using custom-made multibranched endografts. J Endovasc Ther. 2013;20:672-7.

22. Bisdas T, Donas KP, Bosiers MJ, Torsello G, Austermann M. Custom-made versus off-the-shelf multibranched endografts for endovascular repair of thoracoabdominal aortic aneurysms. J Vasc Surg. 2014;60:1186-95.

23. Criado FJ, McKendrick C, Criado FR. Technical solutions for common problems in TEVAR: managing access and aortic branches. J Endovasc Ther. 2009;16(suppl 1):I63-79.

24. Rockman C. Reducing complications by better case selection: anatomic considerations. Semin Vasc Surg. 2004;17:298-306.

25. Garcia-Toca M, Eskandari MK. Regulatory TEVAR clinical trials. J Vasc Surg. 2010;52(4 suppl):22S-5S.

26. Fernandez JD, Craig JM, Garrett HE Jr, et al. Endovascular management of iliac rupture during endovascular aneurysm repair. J Vasc Surg. 2009;50:1293-300.

27. Arnaoutakis GJ, Schneider EB, Arnaoutakis DJ, et al. Influence of gender on outcomes after thoracic endovascular aneurysm repair. J Vasc Surg. 2014;59:45-51.

28. Lee WA, Berceli SA, Huber TS, et al. Morbidity with retroperitoneal procedures during endovascular abdominal aortic aneurysm repair. J Vasc Surg. 2003;38:459-63. discussion 464-5.

29. Melissano G, Tshomba Y, Rinaldi E, Chiesa R. Initial clinical experience with a new low-profile thoracic endograft. J Vasc Surg. 2015;62(2):336-42. https:// doi.org/10.1016/j.jvs.2015.02.049.

30. Torsello GF, Inchingolo M, Austermann M, Torsello GB, Panuccio G, Bisdas T. Durability of a low-profile stent graft for thoracic endovascular aneurysm repair. J Vasc Surg. 2017;66(6):1638-43.

31. Ramanan B, Fernandez CC, Sobel JD, Gasper WJ, Vartanian SM, Reilly LM, Chuter TA, Hiramoto JS. Low-profile versus standard-profile multibranched thoracoabdominal aortic stent grafts. J Vasc Surg. 2016;64(1):39-45.

32. Maurel B, Delclaux N, Sobocinski J, Hertault A, Martin-Gonzalez T, Moussa M, Spear R, Le Roux M, Azzaoui R, Tyrrell M, Haulon S. The impact of early pelvic and lower limb reperfusion and attentive peri-operative management on the incidence of spinal cord ischemia during thoracoabdominal aortic aneurysm endovascular repair. Eur J Vasc Endovasc Surg. 2015;49(3):248-54.

33. de Souza LR, Oderich GS, Banga PV, Hofer JM, Wigham JR, Cha S, Gloviczki P. Outcomes of total percutaneous endovascular aortic repair for thoracic, fenestrated, and branched endografts. J Vasc Surg. 2015;62(6):1442.e3-9.e3. 
34. Derrow AE, Seeger JM, Dame DA, Carter RL, Ozaki CK, Flynn TC, Huber TS. The outcome in the United States after thoracoabdominal aortic aneurysm repair, renal artery bypass, and mesenteric revascularization. J Vasc Surg. 2001;34(1):54-61.

35. Conrad MF, Crawford RS, Davison JK, Cambria RP. Thoracoabdominal aneurysm repair: a 20-year perspective. Ann Thorac Surg. 2007;83(2):S856-61. discussion S890-2.

36. Coselli JS, Bozinovski J, LeMaire SA. Open surgical repair of 2286 thoracoabdominal aortic aneurysms. Ann Thorac Surg. 2007;83(2):S862-4. discussion S890-2.

37. Svensson LG, Crawford ES, Hess KR, Coselli JS, Safi HJ. Experience with 1509 patients undergoing thoracoabdominal aortic operations. J Vasc Surg. 1993;17(2):357-68. discussion 368-70.

38. Bensley RP, Curran T, Hurks R, Lo RC, Wyers MC, Hamdan AD, Chaikof EL, Schermerhorn ML. Open repair of intact thoracoabdominal aortic aneurysms in the American College of Surgeons National Surgical Quality Improvement Program. J Vasc Surg. 2013;58(4):894-900.

39. Tshomba Y, Kahlberg A, Melissano G, Coppi G, Marone E, Ferrari D, Lembo R, Chiesa R. Comparison of renal perfusion solutions during thoracoabdominal aortic aneurysm repair. J Vasc Surg. 2014;59(3):623-33.

40. Chuter TA, Gordon RL, Reilly LM, Goodman JD, Messina LM. An endovascular system for thoracoabdominal aortic aneurysm repair. J EndovascTher. 2001;8(1):25-33.

41. Greenberg R, Eagleton M, Mastracci T. Branched endografts for thoracoabdominal aneurysms. J Thorac Cardiovasc Surg. 2010;140(6 Suppl):S171-8.

42. Eagleton MJ, Follansbee M, Wolski K, Mastracci T, Kuramochi Y. Fenestrated and branched endovascular aneurysm repair outcomes for type II and III thoracoabdominal aortic aneurysms. J Vasc Surg. 2016;63(4):930-42.

43. Oderich GS, Ribeiro M, Reis de Souza L, Hofer J, Wigham J, Cha S. Endovascular repair of thoracoabdominal aortic aneurysms using fenestrated and branched endografts. J Thorac Cardiovasc Surg. 2017;153(2):S32.e7-41.e7.

44. Verzini F, Loschi D, De Rango P, Ferrer C, Simonte G, Coscarella C, Pogany G, Cao P. Current results of total endovascular repair of thoracoabdominal aortic aneurysms. J Cardiovasc Surg. 2014;55:9-19.

45. Verhoeven EL, Katsargyris A, Bekkema F, Oikonomou K, Zeebregts CJ, Ritter W, Tielliu IF. Ten-year experience with endovascular repair of thoracoabdominal aortic aneurysms: results from 166 consecutive patients. Eur J Vasc Endovasc Surg. 2015;49(5):524-31.

46. Oikonomou K, Kopp R, Katsargyris A, Pfister K, Verhoeven EL, Kasprzak P. Outcomes of fenestrated/ branched endografting in post-dissection thoracoabdominal aortic aneurysms. Eur J Vasc Endovasc Surg. 2014;48(6):641-8.
47. Reilly LM, Rapp JH, Grenon SM, Hiramoto JS, Sobel J, Chuter TA. Efficacy and durability of endovascular thoracoabdominal aortic aneurysm repair using the caudally directed cuff technique. J Vasc Surg. 2012;56(1):53-63. discussion 63-4.

48. Mastracci TM, Greenberg RK, Eagleton MJ, Hernandez AV. Durability of branches in branched and fenestrated endografts. J Vasc Surg. 2013;57(4):92633. https://doi.org/10.1016/j.jvs.2012.09.071.

49. Beach JM, Rajeswaran J, Parodi FE, Kuramochi Y, Brier C, Blackstone E, Eagleton MJ. Survival affects decision making for fenestrated and branched endovascular aortic repair. J Vasc Surg. 2017;67(3):722. e8-34.e8.

50. Lobato AC, Camacho-Lobato L. Endovascular treatment of complex aortic aneurysms using the sandwich technique. J Endovasc Ther. 2012;19: 691-706.

51. Lobato AC. Sandwich technique for aortoiliac aneurysms extending to the internal iliac artery or isolated common/internal iliac artery aneurysms: a new endovascular approach to preserve pelvic circulation. J Endovasc Ther. 2011;18:106-11.

52. Yoshida R, Yoshida WB, Kolvenbach R, Vieira PR. Modified "stent-graft sandwich" technique for treatment of isolated common iliac artery aneurysm in patient with Marfan syndrome. Ann Vasc Surg. 2012;26:419.

53. Kolvenbach RR, Yoshida R, Pinter L, Zhu Y, Lin F. Urgent endovascular treatment of thoraco-abdominal aneurysms using a sandwich technique and chimney graftsda technical description. Eur J Vasc Endovasc Surg. 2011;41:54-60.

54. Schwierz E, Kolvenbach RR, Yoshida R, Yoshida $\mathrm{W}$. Experience with the sandwich technique in endovascular thoraco- abdominal aortic aneurysm repair. J Vasc Surg. 2014;59:1562e9.

55. de Bruin JL, Yeung KK, Niepoth WW, Lely RJ, Cheung Q, de Vries A, et al. Geometric study of various chimney graft configurations in an in vitro juxtarenal aneurysm model. $J$ Endovasc Ther. 2013;20:184-90.

56. Niepoth WW, de Bruin JL, Yeung KK, Lely RJ, Devrome AN, Wisselink W, et al. A proof-of-concept in vitro study to determine if EndoAnchors can reduce gutter size in chimney graft configurations. J Endovasc Ther. 2013;20:498-505.

57. Makaroun MS, Dillavou ED, Kee ST, Sicard G, Chaikof E, Bavaria J, Williams D, Cambria RP, Mitchell RS. Endovascular treatment of thoracic aortic aneurysms: results of the phase II multicenter trial of the GORE TAG thoracic endoprosthesis. J Vasc Surg. 2005;41(1):1-9.

58. Bavaria JE, Appoo JJ, Makaroun MS, Verter J, Yu ZF, Mitchell RS, Gore TAG Investigators. Endovascular stent grafting versus open surgical repair of descending thoracic aortic aneurysms in low-risk patients: a multicenter comparative trial. J Thorac Cardiovasc Surg. 2007;133(2):369-77. 
59. Matsumura JS, Cambria RP, Dake MD, Moore RD, Svensson LG, Snyder S, TX2 Clinical Trial Investigators. International controlled clinical trial of thoracic endovascular aneurysm repair with the Zenith TX2 endovascular graft: 1-year results. J Vasc Surg. 2008;47(2):247-57. discussion 257.

60. Fairman RM, Criado F, Farber M, Kwolek C, Mehta M, White R, Lee A, Tuchek JM, VALOR Investigators. Pivotal results of the medtronic vascular talent thoracic stent graft system: the VALOR trial. J Vasc Surg. 2008;48(3):546-54.

61. Greenberg RK, Lu Q, Roselli EE, Svensson LG, Moon MC, Hernandez AV, Dowdall J, Cury M, Francis C, Pfaff K, Clair DG, Ouriel K, Lytle BW. Contemporary analysis of descending thoracic and thoracoabdominal aneurysm repair: a comparison of endovascular and open techniques. Circulation. 2008;118(8):808-17. https://doi.org/10.1161/ CIRCULATIONAHA.108.769695.

62. Ferrer C, Cao P, De Rango P, Tshoma Y, Verzini F, Melissano $\mathrm{G}$, et al. A propensity-matched comparison for endovascular and open repair of thoracoabdominal aortic aneurysms. J Vasc Surg. 2016;63:1201e7.

63. Austermann M, Donas KP, Panuccio G, Troisi N, Torsello G. Pararenal and thoracoabdominal aortic aneurysm repair with fenestrated and branched endografts: lessons learned and future directions. J Endovasc Ther. 2011;18:157-60.

64. Haulon S, Greenberg RK. Part two: treatment of type IV thoracoabdominal aneurysmsd fenestrated stent-graft repair is now the best option. Eur J Vasc Endovasc Surg. 2011;42:4-8.

65. Troisi N, Donas KP, Austermann M, Tessarek J, Umscheid T, Torsello G. Secondary procedures after aortic aneurysm repair with fenestrated and branched endografts. J Endovasc Ther. 2011;18:146-53.

66. Raux M, Patel VI, Cochennec F, Mukhopadhyay $\mathrm{S}$, Desgranges P, Cambria RP, et al. A propensitymatched comparison of outcomes for fenestrated endovascular aneurysm repair and open surgical repair of complex abdominal aortic aneurysms. J Vasc Surg. 2014;60:858-63. 